$\mathrm{J}$ o u r n a l of

Mathematics

and Applications

JMA No 38, pp 151-169 (2015)

\title{
Structure of solutions of nonautonomous optimal control problems in metric spaces
}

\author{
Alexander J. Zaslavski
}

\begin{abstract}
We establish turnpike results for a nonautonomous discrete-time optimal control system describing a model of economic dynamics.
\end{abstract}

AMS Subject Classification: 49J99, 91B55, 91B62

Keywords and Phrases: Compact metric space, good program, infinite horizon problem, overtaking optimal program, turnpike property

\section{Introduction}

The study of the existence, the structure and properties of (approximate) solutions of optimal control problems defined on infinite intervals and on sufficiently large intervals has recently been a rapidly growing area of research $[4-8,10,11,14,15$, $16,18-20,22,23,27,30]$. These problems arise in engineering $[1,32]$, in models of economic growth $[2,9,12,17,21,24,25,27-29,31]$, in infinite discrete models of solid-state physics related to dislocations in one-dimensional crystals [3, 26] and in the theory of thermodynamical equilibrium for materials $[13,16]$.

In this paper we study the structure of approximate solutions of nonautonomous discrete-time optimal control systems arising in economic dynamics which are determined by sequences of lower semicontinuous objective functions.

For each nonempty set $Y$ denote by $\mathcal{B}(Y)$ the set of all bounded functions $f: Y \rightarrow$ $R^{1}$ and for each $f \in \mathcal{B}(Y)$ set

$$
\|f\|=\sup \{|f(y)|: y \in Y\} .
$$

For each nonempty compact metric space $Y$ denote by $C(Y)$ the set of all continuous functions $f: Y \rightarrow R^{1}$.

COPYRIGHT (c) by Publishing Department Rzeszów University of Technology P.O. Box 85, 35-959 Rzeszów, Poland 
Let $(X, \rho)$ be a compact metric space with the metric $\rho$. The set $X \times X$ is equipped with the metric $\rho_{1}$ defined by

$$
\rho_{1}\left(\left(x_{1}, x_{2}\right),\left(y_{1}, y_{2}\right)\right)=\rho\left(x_{1}, y_{1}\right)+\rho\left(x_{2}, y_{2}\right),\left(x_{1}, x_{2}\right),\left(y_{1}, y_{2}\right) \in X \times X \text {. }
$$

For each integer $t \geq 0$ let $\Omega_{t}$ be a nonempty closed subset of the metric space $X \times X$.

Let $T \geq 0$ be an integer. A sequence $\left\{x_{t}\right\}_{t=T}^{\infty} \subset X$ is called a program if $\left(x_{t}, x_{t+1}\right) \in \Omega_{t}$ for all integers $t \geq T$.

Let $T_{1}, T_{2}$ be integers such that $0 \leq T_{1}<T_{2}$. A sequence $\left\{x_{t}\right\}_{t=T_{1}}^{T_{2}} \subset X$ is called a program if $\left(x_{t}, x_{t+1}\right) \in \Omega_{t}$ for all integers $t$ satisfying $T_{1} \leq t<T_{2}$.

We assume that there exists a program $\left\{x_{t}\right\}_{t=0}^{\infty}$. Denote by $\mathcal{M}$ the set of all sequences of functions $\left\{f_{t}\right\}_{t=0}^{\infty}$ such that for each integer $t \geq 0$

$$
f_{t} \in \mathcal{B}\left(\Omega_{t}\right)
$$

and that

$$
\sup \left\{\left\|f_{t}\right\|: t=0,1, \ldots\right\}<\infty .
$$

For each pair of sequences $\left\{f_{t}\right\}_{t=0}^{\infty},\left\{g_{t}\right\}_{t=0}^{\infty} \in \mathcal{M}$ set

$$
d\left(\left\{f_{t}\right\}_{t=0}^{\infty},\left\{g_{t}\right\}_{t=0}^{\infty}\right)=\sup \left\{\left\|f_{t}-g_{t}\right\|: t=0,1, \ldots\right\} .
$$

It is easy to see that $d: \mathcal{M} \times \mathcal{M} \rightarrow[0, \infty)$ is a metric on $\mathcal{M}$ and that the metric space $(\mathcal{M}, d)$ is complete.

Let $\left\{f_{t}\right\}_{t=0}^{\infty} \in \mathcal{M}$. We consider the following optimization problems

$$
\begin{gathered}
\sum_{t=T_{1}}^{T_{2}-1} f_{t}\left(x_{t}, x_{t+1}\right) \rightarrow \text { mins. t. }\left\{x_{t}\right\}_{t=T_{1}}^{T_{2}} \text { is a program, } \\
\sum_{t=T_{1}}^{T_{2}-1} f_{t}\left(x_{t}, x_{t+1}\right) \rightarrow \text { min s. t. }\left\{x_{t}\right\}_{t=T_{1}}^{T_{2}} \text { is a program and } x_{T_{1}}=y, \\
\sum_{t=T_{1}}^{T_{2}-1} f_{t}\left(x_{t}, x_{t+1}\right) \rightarrow \text { mins. t. }\left\{x_{t}\right\}_{t=T_{1}}^{T_{2}} \text { is a program and } x_{T_{1}}=y, x_{T_{2}}=z,
\end{gathered}
$$

where $y, z \in X$ and integers $T_{1}, T_{2}$ satisfy $0 \leq T_{1}<T_{2}$.

The interest in these discrete-time optimal problems stems from the study of various optimization problems which can be reduced to this framework, e. g., continuoustime control systems which are represented by ordinary differential equations whose cost integrand contains a discounting factor [12], the study of the discrete FrenkelKontorova model related to dislocations in one-dimensional crystals $[3,26]$ and the analysis of a long slender bar of a polymeric material under tension in $[13,16]$. Similar optimization problems are also considered in mathematical economics $[9,17,24,28$, 29, 31]. 
For each $y, z \in X$ and each pair of integers $T_{1}, T_{2}$ satisfying $0 \leq T_{1}<T_{2}$ set

$$
\begin{gathered}
U\left(\left\{f_{t}\right\}_{t=0}^{\infty}, T_{1}, T_{2}\right)=\inf \left\{\sum_{t=T_{1}}^{T_{2}-1} f_{t}\left(x_{t}, x_{t+1}\right):\left\{x_{t}\right\}_{t=T_{1}}^{T_{2}} \text { is a program }\right\} \\
U\left(\left\{f_{t}\right\}_{t=0}^{\infty}, T_{1}, T_{2}, y\right)=\inf \left\{\sum_{t=T_{1}}^{T_{2}-1} f_{t}\left(x_{t}, x_{t+1}\right):\left\{x_{t}\right\}_{t=T_{1}}^{T_{2}} \text { is a program and } x_{T_{1}}=y\right\} \\
U\left(\left\{f_{t}\right\}_{t=0}^{\infty}, T_{1}, T_{2}, y, z\right)=\inf \left\{\sum_{t=T_{1}}^{T_{2}-1} f_{t}\left(x_{t}, x_{t+1}\right):\right. \\
\left.\left\{x_{t}\right\}_{t=T_{1}}^{T_{2}} \text { is a program and } x_{T_{1}}=y, x_{T_{2}}=z\right\}
\end{gathered}
$$

Here we assume that the infimum over empty set is $\infty$.

Denote by $\mathcal{M}_{\text {reg }}$ the set of all sequences of functions $\left\{f_{i}\right\}_{i=0}^{\infty} \in \mathcal{M}$ for which there exist a program $\left\{x_{t}^{f}\right\}_{t=0}^{\infty}$ and constants $c_{f}>0, \gamma_{f}>0$ such that the following conditions hold:

(C1) the function $f_{t}$ is lower semicontinuous for all integers $t \geq 0$;

(C2) for each pair of integers $T_{1} \geq 0, T_{2}>T_{1}$,

$$
\sum_{t=T_{1}}^{T_{2}-1} f_{t}\left(x_{t}^{f}, x_{t+1}^{f}\right) \leq U\left(\left\{f_{t}\right\}_{t=0}^{\infty}, T_{1}, T_{2}\right)+c_{f} ;
$$

(C3) for each $\epsilon>0$ there exists $\delta>0$ such that for each integer $t \geq 0$ and each $(x, y) \in \Omega_{t}$ satisfying $\rho\left(x, x_{t}^{f}\right) \leq \delta, \rho\left(y, x_{t+1}^{f}\right) \leq \delta$ we have

$$
\left|f_{t}\left(x_{t}^{f}, x_{t+1}^{f}\right)-f_{t}(x, y)\right| \leq \epsilon
$$

(C4) for each integer $t \geq 0$, each $\left(x_{t}, x_{t+1}\right) \in \Omega_{t}$ satisfying $\rho\left(x_{t}, x_{t}^{f}\right) \leq \gamma_{f}$ and each $\left(x_{t+1}^{\prime}, x_{t+2}^{\prime}\right) \in \Omega_{t+1}$ satisfying $\rho\left(x_{t+2}^{\prime}, x_{t+2}^{f}\right) \leq \gamma_{f}$ there is $x \in X$ such that

$$
\left(x_{t}, x\right) \in \Omega_{t},\left(x, x_{t+2}^{\prime}\right) \in \Omega_{t+1}
$$

moreover, for each $\epsilon>0$ there exists $\delta \in\left(0, \gamma_{f}\right)$ such that for each integer $t \geq$ 0 , each $\left(x_{t}, x_{t+1}\right) \in \Omega_{t}$ and each $\left(x_{t+1}^{\prime}, x_{t+2}^{\prime}\right) \in \Omega_{t+1}$ satisfying $\rho\left(x_{t}, x_{t}^{f}\right) \leq \delta$ and $\rho\left(x_{t+2}^{\prime}, x_{t+2}^{f}\right) \leq \delta$ there is $x \in X$ such that

$$
\left(x_{t}, x\right) \in \Omega_{t},\left(x, x_{t+2}^{\prime}\right) \in \Omega_{t+1}, \rho\left(x, x_{t+1}^{f}\right) \leq \epsilon .
$$

Denote by $\overline{\mathcal{M}}_{\text {reg }}$ the closure of $\mathcal{M}_{\text {reg }}$ in $(\mathcal{M}, d)$. Denote by $\mathcal{M}_{c, \text { reg }}$ the set of all sequences $\left\{f_{i}\right\}_{i=0}^{\infty} \in \mathcal{M}_{\text {reg }}$ such that $f_{i} \in C\left(\Omega_{i}\right)$ for all integers $i \geq 0$ and by $\overline{\mathcal{M}}_{c, r e g}$ the closure of $\mathcal{M}_{c, r e g}$ in $(\mathcal{M}, d)$.

We study the optimization problems stated above with the sequence of objective functions $\left\{f_{i}\right\}_{i=0}^{\infty} \in \mathcal{M}_{\text {reg }}$. Our study is based on the relation between these finite 
horizon problems and the corresponding infinite horizon optimization problem determined by $\left\{f_{i}\right\}_{i=0}^{\infty}$. Note that the condition (C2) means that the program $\left\{x_{t}^{f}\right\}_{t=0}^{\infty}$ is an approximate solution of this infinite horizon problem.

We are interested in turnpike properties of approximate solutions of our optimization problems, which are independent of the length of the interval $T_{2}-T_{1}$, for all sufficiently large intervals. To have these properties means that the approximate solutions of the problems are determined mainly by the objective functions, and are essentially independent of the choice of interval and endpoint conditions, except in regions close to the endpoints. Turnpike properties are well known in mathematical economics. The term was first coined by Samuelson in 1948 (see [26]) where he showed that an efficient expanding economy would spend most of the time in the vicinity of a balanced equilibrium path (also called a von Neumann path).

The paper is organized as follows. In Section 2 we present turnpike results and show the existence of optimal solutions over infinite horizon established in [31]. Our main results (Theorems 3.1 and 3.2) are stated in Section 3. Section 4 contains an example. Our auxiliary results are proved in Section 5. Section 6 contains the proof of Theorem 3.1 while Theorem 3.2 is proved in Section 7 .

\section{Preliminaries} hold.

Let $\left\{f_{i}\right\}_{i=0}^{\infty} \in \mathcal{M}_{\text {reg }}$, a program $\left\{x_{i}^{f}\right\}_{i=0}^{\infty}, c_{f}>0$ and $\gamma_{f}>0$ be such that $(\mathrm{C} 1)-(\mathrm{C} 4)$

In [31] we proved the following useful result.

Proposition 2.1Let $S \geq 0$ be an integer and $\left\{x_{i}\right\}_{i=S}^{\infty}$ be a program. Then either the sequence $\left\{\sum_{i=S}^{T-1} f_{i}\left(x_{i}, x_{i+1}\right)-\sum_{i=S}^{T-1} f_{i}\left(x_{i}^{f}, x_{i+1}^{f}\right)\right\}_{T=S+1}^{\infty}$ is bounded or

$$
\lim _{T \rightarrow \infty}\left[\sum_{i=S}^{T-1} f_{i}\left(x_{i}, x_{i+1}\right)-\sum_{i=S}^{T-1} f_{i}\left(x_{i}^{f}, x_{i+1}^{f}\right)\right]=\infty .
$$

A program $\left\{x_{t}\right\}_{t=S}^{\infty}$, where $S \geq 0$ is an integer, is called $\left(\left\{f_{i}\right\}_{i=0}^{\infty}\right)$-good if the sequence

$$
\left\{\sum_{i=S}^{T-1} f_{i}\left(x_{i}, x_{i+1}\right)-\sum_{i=S}^{T-1} f_{i}\left(x_{i}^{f}, x_{i+1}^{f}\right)\right\}_{T=S+1}^{\infty}
$$

is bounded [9, 27-29, 31].

We say that the sequence $\left\{f_{i}\right\}_{i=0}^{\infty}$ possesses an asymptotic turnpike property (or briefly (ATP)) [31] with $\left\{x_{i}^{f}\right\}_{i=0}^{\infty}$ being the turnpike if for each integer $S \geq 0$ and each $\left(\left\{f_{i}\right\}_{i=0}^{\infty}\right)$-good program $\left\{x_{i}\right\}_{i=S}^{\infty}$,

$$
\lim _{i \rightarrow \infty} \rho\left(x_{i}, x_{i}^{f}\right)=0 .
$$

We say that the sequence $\left\{f_{i}\right\}_{i=0}^{\infty}$ possesses a turnpike property (or briefly (TP)) [31] if for each $\epsilon>0$ and each $M>0$ there exist $\delta>0$ and a natural number $L$ such 
that for each pair of integers $T_{1} \geq 0, T_{2} \geq T_{1}+2 L$ and each program $\left\{x_{t}\right\}_{t=T_{1}}^{T_{2}}$ which satisfies

$$
\sum_{i=T_{1}}^{T_{2}-1} f_{i}\left(x_{i}, x_{i+1}\right) \leq \min \left\{U\left(\left\{f_{i}\right\}_{i=0}^{\infty}, T_{1}, T_{2}, x_{T_{1}}, x_{T_{2}}\right)+\delta, U\left(\left\{f_{i}\right\}_{i=0}^{\infty}, T_{1}, T_{2}\right)+M\right\}
$$

the inequality $\rho\left(x_{i}, x_{i}^{f}\right) \leq \epsilon$ holds for all integers $i=T_{1}+L, \ldots, T_{2}-L$.

The sequence $\left\{x_{i}^{f}\right\}_{i=0}^{\infty}$ is called the turnpike of $\left\{f_{i}\right\}_{i=0}^{\infty}$.

In [31] we proved the following results (see Theorems 2.1-2.4).

Theorem 2.1The sequence $\left\{f_{i}\right\}_{i=0}^{\infty}$ possesses the turnpike property if and only if $\left\{f_{i}\right\}_{i=0}^{\infty}$ possesses (ATP) and the following property:

$(P)$ For each $\epsilon>0$ and each $M>0$ there exist $\delta>0$ and a natural number $L$ such that for each integer $T \geq 0$ and each program $\left\{x_{t}\right\}_{t=T}^{T+L}$ which satisfies

$$
\begin{gathered}
\sum_{i=T}^{T+L-1} f_{i}\left(x_{i}, x_{i+1}\right) \\
\leq \min \left\{U\left(\left\{f_{i}\right\}_{i=0}^{\infty}, T, T+L, x_{T}, x_{T+L}\right)+\delta, U\left(\left\{f_{i}\right\}_{i=0}^{\infty}, T, T+L\right)+M\right\}
\end{gathered}
$$

there is an integer $j \in\{T, \ldots, T+L\}$ for which $\rho\left(x_{j}, x_{j}^{f}\right) \leq \epsilon$.

The property $(\mathrm{P})$ means that if a natural number $L$ is large enough and a program $\left\{x_{t}\right\}_{t=T}^{T+L}$ is an approximate solution of the corresponding finite horizon problem, then there is $j \in\{T, \ldots, T+L\}$ such that $x_{j}$ is close to $x_{j}^{f}$.

We denote by $\operatorname{Card}(A)$ the cardinality of the set $A$.

Theorem 2.2 Assume that the sequence $\left\{f_{i}\right\}_{i=0}^{\infty}$ possesses (ATP) and the property $(P), \epsilon>0$ and $M>0$. Then there exists a natural number $L$ such that for each pair of integers $T_{1} \geq 0, T_{2}>T_{1}+L$ and each program $\left\{x_{t}\right\}_{t=T_{1}}^{T_{2}}$ which satisfies

$$
\sum_{t=T_{1}}^{T_{2}-1} f_{t}\left(x_{t}, x_{t+1}\right) \leq U\left(\left\{f_{i}\right\}_{i=0}^{\infty}, T_{1}, T_{2}\right)+M
$$

the following inequality holds:

$$
\operatorname{Card}\left(\left\{t \in\left\{T_{1}, \ldots, T_{2}\right\}: \rho\left(x_{t}, x_{t}^{f}\right)>\epsilon\right\}\right) \leq L
$$

Let $S \geq 0$ be an integer. A program $\left\{x_{t}\right\}_{t=S}^{\infty}$ is called $\left(\left\{f_{i}\right\}_{i=0}^{\infty}\right)$-minimal $[3,26$, 31] if for each integer $T>S$,

$$
\sum_{t=S}^{T-1} f_{t}\left(x_{t}, x_{t+1}\right)=U\left(\left\{f_{i}\right\}_{i=0}^{\infty}, S, T, x_{S}, x_{T}\right)
$$


A program $\left\{x_{t}\right\}_{t=S}^{\infty}$ is called $\left(\left\{f_{i}\right\}_{i=0}^{\infty}\right)$-overtaking optimal $[12,27,31]$ if for each program $\left\{x_{t}^{\prime}\right\}_{t=S}^{\infty}$ satisfying $x_{S}=x_{S}^{\prime}$,

$$
\limsup _{T \rightarrow \infty}\left(\sum_{t=S}^{T-1} f_{t}\left(x_{t}, x_{t+1}\right)-\sum_{t=S}^{T-1} f_{t}\left(x_{t}^{\prime}, x_{t+1}^{\prime}\right)\right) \leq 0
$$

Theorem 2.3 Assume that the sequence $\left\{f_{i}\right\}_{i=0}^{\infty}$ possesses (ATP), $z \in X, S \geq 0$ is an integer and that there exists an $\left(\left\{f_{i}\right\}_{i=0}^{\infty}\right)$-good program $\left\{x_{t}\right\}_{t=S}^{\infty}$ satisfying $x_{S}=z$. Then there exists an $\left(\left\{f_{i}\right\}_{i=0}^{\infty}\right)$-overtaking optimal program $\left\{x_{t}^{*}\right\}_{t=S}^{\infty}$ satisfying $x_{S}^{*}=z$.

Theorem 2.4 Assume that the sequence $\left\{f_{i}\right\}_{i=0}^{\infty}$ possesses (ATP), $z \in X, S \geq 0$ is an integer and that there exists an $\left(\left\{f_{i}\right\}_{i=0}^{\infty}\right)$-good program $\left\{\bar{x}_{t}\right\}_{t=S}^{\infty}$ satisfying $\bar{x}_{S}=z$. Let a program $\left\{x_{t}\right\}_{t=S}^{\infty}$ satisfy $x_{S}=z$. Then the following properties are equivalent.

(i) $\left\{x_{t}\right\}_{t=S}^{\infty}$ is an $\left(\left\{f_{i}\right\}_{i=0}^{\infty}\right)$-overtaking optimal program;

(ii) the program $\left\{x_{t}\right\}_{t=S}^{\infty}$ is $\left(\left\{f_{i}\right\}_{i=0}^{\infty}\right)$-minimal and $\left(\left\{f_{i}\right\}_{i=0}^{\infty}\right)$-good;

(iii) the program $\left\{x_{t}\right\}_{t=S}^{\infty}$ is $\left(\left\{f_{i}\right\}_{i=0}^{\infty}\right)$-minimal and satisfies $\lim _{t \rightarrow \infty} \rho\left(x_{t}, x_{t}^{f}\right)=0$.

\section{Main results} hold.

Let $\left\{f_{i}\right\}_{i=0}^{\infty} \in \mathcal{M}_{\text {reg }}$, a program $\left\{x_{i}^{f}\right\}_{i=0}^{\infty}, c_{f}>0$ and $\gamma_{f}>0$ be such that $(\mathrm{C} 1)-(\mathrm{C} 4)$

We say that the sequence $\left\{f_{i}\right\}_{i=0}^{\infty}$ possesses a strong asymptotic turnpike property (or briefly (SATP)) with $\left\{x_{i}^{f}\right\}_{i=0}^{\infty}$ being the turnpike if for each integer $S \geq 0$ and each $\left(\left\{f_{i}\right\}_{i=0}^{\infty}\right)$-good program $\left\{x_{i}\right\}_{i=S}^{\infty}$,

$$
\sum_{i=0}^{\infty} \rho\left(x_{i}, x_{i}^{f}\right)<\infty
$$

Clearly, (SATP) implies (ATP).

We say that the sequence $\left\{f_{i}\right\}_{i=0}^{\infty}$ possesses a a strong turnpike property (or briefly (STP)) if for each $\epsilon>0$ and each $M>0$ there exist $\delta>0$ and a natural number $L$ such that for each pair of integers $T_{1} \geq 0, T_{2} \geq T_{1}+2 L$ and each program $\left\{x_{t}\right\}_{t=T_{1}}^{T_{2}}$ which satisfies

$$
\sum_{i=T_{1}}^{T_{2}-1} f_{i}\left(x_{i}, x_{i+1}\right) \leq \min \left\{U\left(\left\{f_{i}\right\}_{i=0}^{\infty}, T_{1}, T_{2}, x_{T_{1}}, x_{T_{2}}\right)+\delta, U\left(\left\{f_{i}\right\}_{i=0}^{\infty}, T_{1}, T_{2}\right)+M\right\}
$$

the inequality $\sum_{i=T_{1}+L}^{T_{2}-L} \rho\left(x_{i}, x_{i}^{f}\right) \leq \epsilon$ holds.

The sequence $\left\{x_{i}^{f}\right\}_{i=0}^{\infty}$ is called the turnpike of $\left\{f_{i}\right\}_{i=0}^{\infty}$.

Clearly, (STP) implies (TP). 
In this paper we prove the following two results which are extensions of Theorems 2.1 and 2.2 respectively.

Theorem 3.1 The sequence $\left\{f_{i}\right\}_{i=0}^{\infty}$ possesses the strong turnpike property if and only if $\left\{f_{i}\right\}_{i=0}^{\infty}$ possesses $(S A T P)$ and the property $(P)$.

Theorem 3.2 Assume that the sequence $\left\{f_{i}\right\}_{i=0}^{\infty}$ possesses (SATP) and the property $(P)$, and $M>0$. Then there exist a natural number $L$ and $M_{0}>0$ such that for each pair of integers $T_{1} \geq 0, T_{2}>T_{1}+L$ and each program $\left\{x_{t}\right\}_{t=T_{1}}^{T_{2}}$ which satisfies

$$
\sum_{t=T_{1}}^{T_{2}-1} f_{t}\left(x_{t}, x_{t+1}\right) \leq U\left(\left\{f_{i}\right\}_{i=0}^{\infty}, T_{1}, T_{2}\right)+M
$$

the following inequality holds:

$$
\sum_{i=T_{1}}^{T_{2}} \rho\left(x_{i}, x_{i}^{f}\right) \leq M_{0}
$$

\section{An example} hold.

Let $\left\{f_{i}\right\}_{i=0}^{\infty} \in \mathcal{M}_{r e g}$, a program $\left\{x_{i}^{f}\right\}_{i=0}^{\infty}, c_{f}>0$ and $\gamma_{f}>0$ be such that $(\mathrm{C} 1)-(\mathrm{C} 4)$

Now we show that $\left\{f_{i}\right\}_{i=0}^{\infty}$ is approximated by elements of $\mathcal{M}_{\text {reg }}$ possessing (STP). For each $r \in(0,1)$ and all integers $i \geq 0$ set

$$
f_{i}^{(r)}(x, y)=f_{i}(x, y)+r \rho\left(x, x_{i}^{f}\right),(x, y) \in \Omega_{i}
$$

Clearly, $\left\{f_{i}^{(r)}\right\}_{i=0}^{\infty} \in \mathcal{M}_{r e g}$ for all $r \in(0,1)$ and $\lim _{r \rightarrow 0^{+}} d\left(\left\{f_{i}^{(r)}\right\}_{i=0}^{\infty},\left\{f_{i}\right\}_{i=0}^{\infty}\right)=0$.

Proposition 4.1 Let $r \in(0,1)$. Then $\left\{f_{i}^{(r)}\right\}_{i=0}^{\infty}$ possesses $(S T P)$ with $\left\{x_{i}^{f}\right\}_{i=0}^{\infty}$ being the turnpike.

Proof. By Proposition 2.6 of [31], $\left\{f_{i}^{(r)}\right\}_{i=0}^{\infty}$ possesses (TP) with $\left\{x_{i}^{f}\right\}_{i=0}^{\infty}$ being the turnpike. It follows from Theorem 2.1 that $\left\{f_{i}^{(r)}\right\}_{i=0}^{\infty}$ has the property $(\mathrm{P})$. In view of Theorem 3.1 it is sufficient to show that $\left\{f_{i}^{(r)}\right\}_{i=0}^{\infty}$ possesses (SATP).

Assume that $S \geq 0$ is an integer and that a program $\left\{x_{i}\right\}_{i=S}^{\infty}$ is $\left(\left\{f_{i}^{(r)}\right\}_{i=0}^{\infty}\right)$-good. Then there is $c_{1}>0$ such that

$$
\left|\sum_{t=S}^{T-1} f_{t}^{(r)}\left(x_{t}, x_{t+1}\right)-\sum_{t=S}^{T-1} f_{t}^{(r)}\left(x_{t}^{f}, x_{t+1}^{f}\right)\right| \leq c_{1} \text { forallintegers } T>S
$$

By Proposition 2.1, (4.1) and (4.2), $\sum_{t=S}^{\infty} \rho\left(x_{t}, x_{t}^{f}\right)<\infty$. Thus (SATP) holds. Proposition 4.1 is proved. 


\section{$5 \quad$ Auxiliary results}

We use the notation, definitions and assumptions introduced in Sections 1-3.

The following two results were obtained in [31].

Lemma 5.1 Let an integer $S \geq 0$ and a program $\left\{x_{i}\right\}_{i=S}^{\infty}$ be $\left(\left\{f_{i}\right\}_{t=0}^{\infty}\right)$-good. Then there is a number $c>0$ such that for each pair of integers $T_{1} \geq S$ and $T_{2}>T_{1}$,

$$
\sum_{i=T_{1}}^{T_{2}-1} f_{i}\left(x_{i}, x_{i+1}\right) \leq U\left(\left\{f_{i}\right\}_{i=0}^{\infty}, T_{1}, T_{2}\right)+c
$$

and the following property holds:

for each $\epsilon>0$ there exists a natural number $L$ such that for each integer $T_{1} \geq L$ and each integer $T_{2}>T_{1}$,

$$
\sum_{i=T_{1}}^{T_{2}-1} f_{i}\left(x_{i}, x_{i+1}\right) \leq U\left(\left\{f_{i}\right\}_{i=0}^{\infty}, T_{1}, T_{2}, x_{T_{1}}, x_{T_{2}}\right)+\epsilon .
$$

Lemma 5.2 Let $\epsilon>0$. Then there exists $\delta>0$ such that for each pair of integers $T_{1}>0, T_{2}>T_{1}+2$ and each program $\left\{x_{i}\right\}_{i=T_{1}}^{T_{2}}$ satisfying

$$
\begin{gathered}
\rho\left(x_{T_{1}+1}, x_{T_{1}+1}^{f}\right) \leq \delta, \rho\left(x_{T_{2}-1}, x_{T_{2}-1}^{f}\right) \leq \delta \\
\sum_{i=T_{1}+1}^{T_{2}-2} f_{i}\left(x_{i}, x_{i+1}\right) \leq U\left(\left\{f_{i}\right\}_{i=0}^{\infty}, T_{1}+1, T_{2}-1, x_{T_{1}+1}, x_{T_{2}-1}\right)+\delta
\end{gathered}
$$

there exists a program $\left\{\tilde{x}_{i}\right\}_{i=T_{1}-1}^{T_{2}+1}$ such that

$$
\tilde{x}_{T_{1}-1}=x_{T_{1}-1}^{f}, \tilde{x}_{T_{2}+1}=x_{T_{2}+1}^{f}, \tilde{x}_{i}=x_{i}, i=T_{1}+1, \ldots T_{2}-1
$$

and that the following inequality holds:

$$
\sum_{i=T_{1}-1}^{T_{2}} f_{i}\left(\tilde{x}_{i}, \tilde{x}_{i+1}\right) \leq \sum_{i=T_{1}-1}^{T_{2}} f_{i}\left(x_{i}^{f}, x_{i+1}^{f}\right)+\epsilon .
$$

Lemma 5.3 Assume that $\left\{f_{i}\right\}_{i=0}^{\infty}$ possesses (SATP) and let $\epsilon>0$. Then there exist $\delta>0$ and a natural number $L$ such that for each pair of integers $T_{2}>T_{1} \geq L$ and each program $\left\{x_{i}\right\}_{i=T_{1}}^{T_{2}}$ satisfying

$$
x_{T_{1}}=x_{T_{1}}^{f}, x_{T_{2}}=x_{T_{2}}^{f}, \sum_{i=T_{1}}^{T_{2}-1} f_{i}\left(x_{i}, x_{i+1}\right) \leq \sum_{i=T_{1}}^{T_{2}-1} f_{i}\left(x_{i}^{f}, x_{i+1}^{f}\right)+\delta
$$

the inequality $\sum_{t=T_{1}}^{T_{2}} \rho\left(x_{t}, x_{t}^{f}\right) \leq \epsilon$ holds. 
Proof. Assume that the lemma is not true. Then there exist sequences of natural numbers $\left\{T_{k}\right\}_{k=1}^{\infty},\left\{S_{k}\right\}_{k=1}^{\infty}$ such that for each natural number $k$,

$$
T_{k}<S_{k}<T_{k+1}
$$

and there exists a program $\left\{x_{i}^{(k)}\right\}_{i=T_{k}}^{S_{k}}$ such that

$$
\begin{gathered}
x_{T_{k}}^{(k)}=x_{T_{k}}^{f}, x_{S_{k}}^{(k)}=x_{S_{k}}^{f}, \\
\sum_{i=T_{k}}^{S_{k}-1} f_{i}\left(x_{i}^{(k)}, x_{i+1}^{(k)}\right) \leq \sum_{i=T_{k}}^{S_{k}-1} f_{i}\left(x_{i}^{f}, x_{i+1}^{f}\right)+2^{-k}, \\
\sum_{i=T_{k}}^{S_{k}} \rho\left(x_{i}^{(k)}, x_{i}^{f}\right)>\epsilon .
\end{gathered}
$$

Define a sequence $\left\{x_{i}\right\}_{i=0}^{\infty} \subset X$ as follows: for each integer $k \geq 1$,

$$
\begin{gathered}
\qquad x_{i}=x_{i}^{(k)}, i=T_{k}, \ldots, S_{k}, \\
x_{i}=x_{i}^{f} \text { for all integers } i \geq 0 \text { such that } i \notin \cup_{k=1}^{\infty}\left\{T_{k}, \ldots, S_{k}\right\} .
\end{gathered}
$$

By (5.1), (5.4) and (5.5) $\left\{x_{i}\right\}_{i=0}^{\infty}$ is a well-defined program. By (5.2), (5.4) and (5.5) for each integer $p \geq 1$,

$$
\sum_{i=0}^{S_{p}} f_{i}\left(x_{i}, x_{i+1}\right) \leq \sum_{i=0}^{S_{p}} f_{i}\left(x_{i}^{f}, x_{i+1}^{f}\right)+\sum_{i=1}^{p} 2^{-i} .
$$

Combined with Proposition 2.1 this implies that the program $\left\{x_{i}\right\}_{i=0}^{\infty}$ is $\left(\left\{f_{i}\right\}_{i=0}^{\infty}\right)$ good. In view of (SATP),

$$
\sum_{i=0}^{\infty} \rho\left(x_{i}, x_{i}^{f}\right)<\infty .
$$

On the other hand, it follows from (5.3), (5.4) and (5.5) that

$$
\sum_{i=0}^{\infty} \rho\left(x_{i}, x_{i}^{f}\right) \geq \sum_{k=0}^{\infty}\left(\sum_{i=T_{k}}^{S_{k}} \rho\left(x_{i}^{(k)}, x_{i}^{f}\right)\right) \geq \sum_{k=0}^{\infty} \epsilon=\infty .
$$

The contradiction we have reached completes the proof of Lemma 5.3.

Lemma 5.4 Assume that $\left\{f_{i}\right\}_{i=0}^{\infty}$ possesses (SATP) and let $\epsilon>0$. Then there exist $\delta>0$ and a natural number $L$ such that for each pair of integers $T_{1}, T_{2}$ satisfying $T_{1}>L, T_{2}>T_{1}+2$ and each program $\left\{x_{i}\right\}_{i=T_{1}}^{T_{2}}$ satisfying

$$
\rho\left(x_{T_{1}+1}, x_{T_{1}+1}^{f}\right) \leq \delta, \rho\left(x_{T_{2}-1}, x_{T_{2}-1}^{f}\right) \leq \delta,
$$




$$
\sum_{i=T_{1}+1}^{T_{2}-2} f_{i}\left(x_{i}, x_{i+1}\right) \leq U\left(\left\{f_{i}\right\}_{i=0}^{\infty}, T_{1}+1, T_{2}-1, x_{T_{1}+1}, x_{T_{2}-1}\right)+\delta
$$

the following inequality holds:

$$
\sum_{t=T_{1}+1}^{T_{2}-1} \rho\left(x_{t}, x_{t}^{f}\right) \leq \epsilon .
$$

Proof. By Lemma 5.3, there exist $\delta_{1}>0$ and a natural number $L$ such that for each pair of integers $S_{2}>S_{1} \geq L$ and each program $\left\{x_{i}\right\}_{i=S_{1}}^{S_{2}}$ satisfying

$$
x_{S_{i}}=x_{S_{i}}^{f}, i=1,2, \sum_{i=S_{1}}^{S_{2}-1} f_{i}\left(x_{i}, x_{i+1}\right) \leq \sum_{i=S_{1}}^{S_{2}-1} f_{i}\left(x_{i}^{f}, x_{i+1}^{f}\right)+\delta_{1}
$$

we have

$$
\sum_{i=S_{1}}^{S_{2}} \rho\left(x_{i}, x_{i}^{f}\right) \leq \epsilon .
$$

By Lemma 5.2 there exist $\delta>0$ such that for each pair of integers $T_{1}>0, T_{2}>T_{1}+2$ and each program $\left\{x_{i}\right\}_{i=T_{1}}^{T_{2}}$ satisfying

$$
\begin{gathered}
\rho\left(x_{T_{1}+1}, x_{T_{1}+1}^{f}\right) \leq \delta, \rho\left(x_{T_{2}-1}, x_{T_{2}-1}^{f}\right) \leq \delta \\
\sum_{i=T_{1}+1}^{T_{2}-2} f_{i}\left(x_{i}, x_{i+1}\right) \leq U\left(\left\{f_{i}\right\}_{i=0}^{\infty}, T_{1}+1, T_{2}-1, x_{T_{1}+1}, x_{T_{2}-1}\right)+\delta
\end{gathered}
$$

there exists a program $\left\{\tilde{x}_{i}\right\}_{i=T_{1}-1}^{T_{2}+1}$ such that

$$
\begin{gathered}
\tilde{x}_{T_{1}-1}=x_{T_{1}-1}^{f}, \tilde{x}_{T_{2}+1}=x_{T_{2}+1}^{f}, \tilde{x}_{i}=x_{i}, i=T_{1}+1, \ldots T_{2}-1, \\
\sum_{i=T_{1}-1}^{T_{2}} f_{i}\left(\tilde{x}_{i}, \tilde{x}_{i+1}\right) \leq \sum_{i=T_{1}-1}^{T_{2}} f_{i}\left(x_{i}^{f}, x_{i+1}^{f}\right)+\delta_{1} .
\end{gathered}
$$

Assume that an integer $T_{1}>L$, an integer $T_{2}>T_{1}+2$ and a program $\left\{x_{i}\right\}_{i=T_{1}}^{T_{2}}$ satisfies (5.6) and (5.7). By (5.6), (5.7) and the choice of $\delta$, there exists a program $\left\{\tilde{x}_{i}\right\}_{i=T_{1}-1}^{T_{2}+1}$ which satisfies $(5.10),(5.11)$. By (5.10), (5.11), the choice of $\delta_{1}$ (see (5.8), $(5.9))$,

$$
\sum_{i=T_{1}-1}^{T_{2}+1} \rho\left(\tilde{x}_{i}, x_{i}^{f}\right) \leq \epsilon .
$$

Together with (5.10) this implies that

$$
\sum_{i=T_{1}+1}^{T_{2}-1} \rho\left(x_{i}, x_{i}^{f}\right) \leq \epsilon .
$$

Lemma 5.4 is proved. 


\section{Proof of Theorem 3.1}

Assume that (STP) holds. Then (TP) holds and in view of Theorem 2.1, (ATP) and the the property $(\mathrm{P})$ hold. Therefore

$$
\lim _{t \rightarrow \infty} \rho\left(x_{t}, x_{t}^{f}\right)=0
$$

for each integer $S \geq 0$ and each $\left(\left\{f_{i}\right\}_{i=0}^{\infty}\right)$-good program $\left\{x_{i}\right\}_{i=S}^{\infty}$.

Let us show that (SATP) holds. Assume that $S \geq 0$ is an integer and a program $\left\{x_{i}\right\}_{i=S}^{\infty}$ is $\left(\left\{f_{i}\right\}_{i=0}^{\infty}\right)$-good. Then (6.1) is true. By Lemma 5.1, there is $c>0$ such that for all integers $T_{1} \geq S, T_{2}>T_{1}$,

$$
\sum_{i=T_{1}}^{T_{2}-1} f_{i}\left(x_{i}, x_{i+1}\right) \leq U\left(\left\{f_{i}\right\}_{i=0}^{\infty}, T_{1}, T_{2}\right)+c .
$$

By (STP), there exist $\delta>0$ and a natural number $L_{0}$ such that for each pair of integers $T_{1} \geq 0, T_{2} \geq T_{1}+2 L_{0}$ and each program $\left\{z_{t}\right\}_{t=T_{1}}^{T_{2}}$ which satisfies

$$
\sum_{i=T_{1}}^{T_{2}-1} f_{i}\left(z_{i}, z_{i+1}\right) \leq \min \left\{U\left(\left\{f_{i}\right\}_{i=0}^{\infty}, T_{1}, T_{2}, z_{T_{1}}, z_{T_{2}}\right)+\delta, U\left(\left\{f_{i}\right\}_{i=0}^{\infty}, T_{1}, T_{2}\right)+c\right\}
$$

we have

$$
\sum_{i=T_{1}+L_{0}}^{T_{2}-L_{0}} \rho\left(z_{i}, x_{i}^{f}\right) \leq 1
$$

By Lemma 5.1, there exists a natural number $L_{1}>S$ such that for each integer $T_{1} \geq L_{1}$ and each integer $T_{2}>T_{1}$,

$$
\sum_{i=T_{1}}^{T_{2}-1} f_{i}\left(x_{i}, x_{i+1}\right) \leq U\left(\left\{f_{i}\right\}_{i=0}^{\infty}, T_{1}, T_{2}, x_{T_{1}}, x_{T_{2}}\right)+\delta .
$$

Assume that integers

$$
T_{1} \geq L_{1}, T_{2} \geq T_{1}+2 L_{0}
$$

Then (6.2) and (6.5) hold. In view of (6.2), (6.5), (6.6) and the choice of $\delta, L_{0}$,

$$
\sum_{i=T_{1}+L_{0}}^{T_{2}-L_{0}} \rho\left(x_{i}, x_{i}^{f}\right) \leq 1
$$

Since (6.7) holds for any pair of integers $T_{1}, T_{2}$ satisfying (6.6) we conclude that

$$
\sum_{i=L_{1}+L_{0}}^{\infty} \rho\left(x_{i}, x_{i}^{f}\right) \leq 1 .
$$

This implies that $\sum_{i=0}^{\infty} \rho\left(x_{i}, x_{i}^{f}\right)<\infty$ and that (SATP) holds. Thus we have shown that (STP) implies (SATP) and the property $(\mathrm{P})$. 
Assume that (SATP) and the property (P) hold.

Let $\epsilon>0$ and $M>0$. By Lemma 5.4 there exist $\delta_{0}>0$ and a natural number $L_{0}$ such that for each pair of integers $S_{1}, S_{2}$ satisfying $S_{1}>L_{0}, S_{2}>S_{1}+2$ and each program $\left\{x_{i}\right\}_{i=S_{1}}^{S_{2}}$ satisfying

$$
\begin{gathered}
\rho\left(x_{S_{1}+1}, x_{S_{1}+1}^{f}\right) \leq \delta_{0}, \rho\left(x_{S_{2}-1}, x_{S_{2}-1}^{f}\right) \leq \delta_{0} \\
\sum_{i=S_{1}+1}^{S_{2}-2} f_{i}\left(x_{i}, x_{i+1}\right) \leq U\left(\left\{f_{i}\right\}_{i=0}^{\infty}, S_{1}+1, S_{2}-1, x_{S_{1}+1}, x_{S_{2}-1}\right)+\delta_{0}
\end{gathered}
$$

we have

$$
\sum_{i=S_{1}+1}^{S_{2}-2} \rho\left(x_{i}, x_{i}^{f}\right) \leq \epsilon .
$$

By the property $(\mathrm{P})$ there exist

$$
\delta \in\left(0, \delta_{0}\right)
$$

and a natural number $L_{1}$ such that for each integer $T \geq 0$ and each program $\left\{x_{t}\right\}_{t=T}^{T+L_{1}}$ which satisfies

$$
\begin{gathered}
\sum_{i=T}^{T+L_{1}-1} f_{i}\left(x_{i}, x_{i+1}\right) \\
\leq \min \left\{U\left(\left\{f_{i}\right\}_{i=0}^{\infty}, T, T+L_{1}, x_{T}, x_{T+L_{1}}\right)+\delta, U\left(\left\{f_{i}\right\}_{i=0}^{\infty}, T, T+L_{1}\right)+3 c_{f}+M\right\},
\end{gathered}
$$

there is an integer $j$ such that

$$
j \in\left\{T, \ldots, T+L_{1}\right\}, \rho\left(x_{j}, x_{j}^{f}\right) \leq \delta_{0} .
$$

Choose a natural number

$$
L \geq 4 L_{0}+4 L_{1} .
$$

Assume that a pair of integers $T_{1} \geq 0, T_{2} \geq T_{1}+2 L$ and that a program $\left\{x_{t}\right\}_{t=T_{1}}^{T_{2}}$ satisfies

$$
\sum_{i=T_{1}}^{T_{2}-1} f_{i}\left(x_{i}, x_{i+1}\right) \leq \min \left\{U\left(\left\{f_{i}\right\}_{i=0}^{\infty}, T_{1}, T_{2}, x_{T_{1}}, x_{T_{2}}\right)+\delta, U\left(\left\{f_{i}\right\}_{i=0}^{\infty}, T_{1}, T_{2}\right)+M\right\} .
$$

In order to complete the proof of the theorem it is sufficient to show that

$$
\sum_{i=T_{1}+L}^{T_{2}-L} \rho\left(x_{i}, x_{i}^{f}\right) \leq \epsilon .
$$


Let integers $S_{1}, S_{2}$ satisfy $T_{1}<S_{1}<S_{2}<T_{2}$. By (6.14) and (C2),

$$
\begin{aligned}
& \sum_{i=S_{1}}^{S_{2}-1} f_{i}\left(x_{i}, x_{i+1}\right)=\sum_{i=T_{1}}^{T_{2}-1} f_{i}\left(x_{i}, x_{i+1}\right)-\sum_{i=T_{1}}^{S_{1}-1} f_{i}\left(x_{i}, x_{i+1}\right)-\sum_{i=S_{2}}^{T_{2}-1} f_{i}\left(x_{i}, x_{i+1}\right) \\
& \quad \leq U\left(\left\{f_{i}\right\}_{i=0}^{\infty}, T_{1}, T_{2}\right)+M-U\left(\left\{f_{i}\right\}_{i=0}^{\infty}, T_{1}, S_{1}\right)-U\left(\left\{f_{i}\right\}_{i=0}^{\infty}, S_{2}, T_{2}\right) \\
& \quad \leq \sum_{i=T_{1}}^{T_{2}-1} f_{i}\left(x_{i}^{f}, x_{i+1}^{f}\right)+M-\sum_{i=T_{1}}^{S_{1}-1} f_{i}\left(x_{i}^{f}, x_{i+1}^{f}\right)+c_{f}-\sum_{i=S_{2}}^{T_{2}-1} f_{i}\left(x_{i}^{f}, x_{i+1}^{f}\right)+c_{f} \\
& \quad=\sum_{i=S_{1}}^{S_{2}-1} f_{i}\left(x_{i}^{f}, x_{i+1}^{f}\right)+2 c_{f}+M \leq U\left(\left\{f_{i}\right\}_{i=0}^{\infty}, S_{1}, S_{2}\right)+3 c_{f}+M .
\end{aligned}
$$

Thus

$$
\sum_{i=S_{1}}^{S_{2}-1} f_{i}\left(x_{i}, x_{i+1}\right) \leq U\left(\left\{f_{i}\right\}_{i=0}^{\infty}, S_{1}, S_{2}\right)+3 c_{f}+M
$$

for all pairs of integers $S_{1}, S_{2}$ satisfying $T_{1}<S_{1}<S_{2}<T_{2}$.

By (6.13), (6.14), (6.16), the choice of $\delta$ (see (6.10)-(6.12)) there exist integers

$$
\tau_{1} \in\left\{L_{1}+T_{1}+2 L_{0}, \ldots, T_{1}+2 L_{0}+2 L_{1}\right\}, \tau_{2} \in\left\{T_{2}-2 L_{1}, \ldots, T_{2}-L_{1}\right\}
$$

such that

$$
\rho\left(x_{\tau_{i}}, x_{\tau_{i}}^{f}\right) \leq \delta_{0}, i=1,2
$$

By (6.13) and (6.17),

$$
\tau_{2}-\tau_{1} \geq 2 L_{0}+L
$$

By (6.14) and (6.17),

$$
\sum_{i=\tau_{1}}^{\tau_{2}-1} f_{i}\left(x_{i}, x_{i+1}\right) \leq U\left(\left\{f_{i}\right\}_{i=0}^{\infty}, \tau_{1}, \tau_{2}, x_{\tau_{1}}, x_{\tau_{2}}\right)+\delta .
$$

By (6.19), (6.20), (6.17), (6.18), (6.10) and the choice of $L_{0}$ and $\delta_{0}$ (see (6.7)-(6.9)),

$$
\sum_{i=\tau_{1}}^{\tau_{2}} \rho\left(x_{i}, x_{i}^{f}\right) \leq \epsilon .
$$

Together with (6.13) and (6.17) this implies (6.15). Theorem 3.1 is proved.

\section{$7 \quad$ Proof of Theorem 3.2}

Set

$$
D_{0}=\sup \left\{\rho\left(z_{1}, z_{2}\right): z_{1}, z_{2} \in X\right\} .
$$

We suppose that the sum over empty set is zero. By (SATP), the property $(\mathrm{P})$ and Theorem 3.1, $\left\{f_{i}\right\}_{i=0}^{\infty}$ possesses (STP). By (STP) there exist $\delta \in(0,1)$ and a natural number $L_{0}$ such that the following property holds: 
(a) for each pair of integers $\tau_{1} \geq 0, \tau_{2} \geq \tau_{1}+2 L_{0}$ and each program $\left\{x_{t}\right\}_{t=\tau_{1}}^{\tau_{2}}$ which satisfies

$\sum_{t=\tau_{1}}^{\tau_{2}-1} f_{t}\left(x_{t}, x_{t+1}\right) \leq \min \left\{U\left(\left\{f_{i}\right\}_{i=0}^{\infty}, \tau_{1}, \tau_{2}, x_{\tau_{1}}, x_{\tau_{2}}\right)+\delta, U\left(\left\{f_{i}\right\}_{i=0}^{\infty}, \tau_{1}, \tau_{2}\right)+2 M+4 c_{f}\right\}$

the inequality

$$
\sum_{i=\tau_{1}+L_{0}}^{\tau_{2}-L_{0}} \rho\left(x_{i}, x_{i}^{f}\right) \leq 1
$$

holds.

Choose a natural number

$$
L>\left(4 L_{0}+3\right)\left(\delta^{-1} M+1\right)
$$

and

$$
M_{0}>1+\left(\delta^{-1} M+2\right)\left(1+2 D_{0}\left(2 L_{0}+1\right)\right) .
$$

Assume that integers $T_{1} \geq 0, T_{2}>T_{1}+L$ and that a program $\left\{x_{t}\right\}_{t=T_{1}}^{T_{2}}$ satisfies

$$
\sum_{t=T_{1}}^{T_{2}-1} f_{t}\left(x_{t}, x_{t+1}\right) \leq U\left(\left\{f_{i}\right\}_{i=0}^{\infty}, T_{1}, T_{2}\right)+M
$$

Set

$$
t_{0}=T_{1}
$$

By induction we define a finite strictly increasing sequence of integers $\left\{t_{i}\right\}_{i=0}^{q} \subset$ $\left[T_{1}, T_{2}\right]$ where $q$ is a natural number such that:

$$
t_{q}=T_{2}
$$

(b) for each integer $i$ satisfying $0 \leq i<q-1$,

$$
\sum_{t=t_{i}}^{t_{i+1}-1} f_{t}\left(x_{t}, x_{t+1}\right)>U\left(\left\{f_{i}\right\}_{i=0}^{\infty}, t_{i}, t_{i+1}, x_{t_{i}}, x_{t_{i+1}}\right)+\delta ;
$$

(c) if an integer $i$ satisfies $0 \leq i \leq q-1$ and (7.7), then

$$
t_{i+1}>t_{i}+1 \text { and } \sum_{t=t_{i}}^{t_{i+1}-2} f_{t}\left(x_{t}, x_{t+1}\right) \leq U\left(\left\{f_{j}\right\}_{j=0}^{\infty}, t_{i}, t_{i+1}-1, x_{t_{i}}, x_{t_{i+1}-1}\right)+\delta .
$$

Assume that an integer $p \geq 0$ and we have already defined a strictly increasing sequence of integers $\left\{t_{i}\right\}_{i=0}^{p} \subset\left[T_{1}, T_{2}\right]$ such that $t_{p}<T_{2}$ and that for each integer $i$ satisfying $0 \leq i<p,(7.7)$ and (7.8) hold. (Note that for $p=0$ our assumption holds.) We define $t_{p+1}$. 
There are two cases:

$$
\begin{aligned}
& \sum_{t=t_{p}}^{T_{2}-1} f_{t}\left(x_{t}, x_{t+1}\right) \leq U\left(\left\{f_{i}\right\}_{i=0}^{\infty}, t_{p}, T_{2}, x_{t_{p}}, x_{T_{2}}\right)+\delta \\
& \sum_{t=t_{p}}^{T_{2}-1} f_{t}\left(x_{t}, x_{t+1}\right)>U\left(\left\{f_{i}\right\}_{i=0}^{\infty}, t_{p}, T_{2}, x_{t_{p}}, x_{T_{2}}\right)+\delta .
\end{aligned}
$$

Assume that (7.9) holds. Then we set $q=p+1, t_{q}=T_{2}$, the construction of the sequence is completed and the properties (b), (c) hold.

Assume that (7.10) holds. Set

$$
\begin{gathered}
t_{p+1}=\min \left\{S \in\left\{t_{p}+1, \ldots, T_{2}\right\}:\right. \\
\left.\sum_{t=t_{p}}^{S-1} f_{t}\left(x_{t}, x_{t+1}\right)>U\left(\left\{f_{i}\right\}_{i=0}^{\infty}, t_{p}, S, x_{t_{p}}, x_{S}\right)+\delta\right\} .
\end{gathered}
$$

Clearly, $t_{p+1}$ is well-defined. If $t_{p+1}=T_{2}$, then we set $q=p+1$, the construction is completed and it is not difficult to see that (b) and (c) hold.

Assume that $t_{p+1}<T_{2}$. Then it is easy to see that the assumption made for $p$ is also true for $p+1$.

Clearly our construction is completed after a final number of steps and let $t_{q}=T_{2}$ be its last element, where $q$ is a natural number. It follows from the construction that the properties (b) and (c) hold.

By (7.4) and the property (b)

$$
\begin{aligned}
M & \geq \sum_{t=T_{1}}^{T_{2}-1} f_{t}\left(x_{t}, x_{t+1}\right)-U\left(\left\{f_{i}\right\}_{i=0}^{\infty}, T_{1}, T_{2}\right) \\
& \geq \sum_{t=T_{1}}^{T_{2}-1} f_{t}\left(x_{t}, x_{t+1}\right)-U\left(\left\{f_{i}\right\}_{i=0}^{\infty}, T_{1}, T_{2}, x_{T_{1}}, x_{T_{2}}\right) \\
& \geq \sum\left\{\sum_{t=t_{i}}^{t_{i+1}-1} f_{t}\left(x_{t}, x_{t+1}\right)-U\left(\left\{f_{j}\right\}_{j=0}^{\infty}, t_{i}, t_{i+1}, x_{t_{i}}, x_{t_{i+1}}\right):\right.
\end{aligned}
$$

$i$ is an integer, $0 \leq i<q-1\} \geq \delta(q-1)$,

$$
q \leq \delta^{-1} M+1
$$

Set

$$
A=\left\{i \in\{0, \ldots, q-1\}: t_{i+1}-t_{i}>2 L_{0}\right\} .
$$

Let

$$
j \in A \text {. }
$$


By (b), (c) and (7.13) and (7.14),

$$
\sum_{t=t_{j}}^{t_{j+1}-2} f_{t}\left(x_{t}, x_{t+1}\right) \leq U\left(\left\{f_{i}\right\}_{i=0}^{\infty}, t_{j}, t_{j+1}-1, x_{t_{j}}, x_{t_{j+1}-1}\right)+\delta .
$$

By (7.4), (7.13), (7.14) and (C2),

$$
\begin{aligned}
& \sum_{t=t_{j}}^{t_{j+1}-2} f_{t}\left(x_{t}, x_{t+1}\right)=\sum_{t=T_{1}}^{T_{2}-1} f_{t}\left(x_{t}, x_{t+1}\right) \\
& \quad-\sum\left\{f_{t}\left(x_{t}, x_{t+1}\right): t \text { is an integer, } T_{1} \leq t<t_{j}\right\}-\sum_{t=t_{j+1}-1}^{T_{2}-1} f_{t}\left(x_{t}, x_{t+1}\right) \\
& \leq \sum_{t=T_{1}}^{T_{2}-1} f_{t}\left(x_{t}^{f}, x_{t+1}^{f}\right)+M+c_{f} \\
& \quad-\sum\left\{f_{t}\left(x_{t}^{f}, x_{t+1}^{f}\right): t \text { is an integer, } T_{1} \leq t<t_{j}\right\}+c_{f}-\sum_{t=t_{j+1}-1}^{T_{2}-1} f_{t}\left(x_{t}^{f}, x_{t+1}^{f}\right) \\
& \quad=\sum_{t=t_{j}}^{t_{j+1}-2} f_{t}\left(x_{t}^{f}, x_{t+1}^{f}\right)+M+2 c_{f} \leq U\left(\left\{f_{i}\right\}_{i=0}^{\infty}, t_{j}, t_{j+1}-1\right)+M+3 c_{f} .
\end{aligned}
$$

By (7.13), (7.14), (7.15), (7.16) and property (a),

$$
\sum_{t=t_{j}+L_{0}}^{t_{j+1}-1-L_{0}} \rho\left(x_{t}, x_{t}^{f}\right) \leq 1
$$

for all $j \in A$. By (7.5), (7.6), (7.13), (7.1), (7.17), (7.12) and (7.3),

$$
\begin{aligned}
\sum_{t=0}^{T} \rho\left(x_{t}, x_{t}^{f}\right) \leq & D_{0}+\sum_{j=0}^{q-1}\left(\sum_{t=t_{j}}^{t_{j+1}-1} \rho\left(x_{t}, x_{t}^{f}\right)\right) \\
= & D_{0}+\sum_{j \in A}\left(\sum_{t=t_{j}}^{t_{j+1}-1} \rho\left(x_{t}, x_{t}^{f}\right)\right) \\
& +\sum\left\{\sum_{t=t_{j}}^{t_{j+1}-1} \rho\left(x_{t}, x_{t}^{f}\right): j \in\{0, \ldots, q-1\} \backslash A\right\} \\
\leq & D_{0}+\sum_{j \in A}\left(L_{0} D_{0}+1+D_{0}\left(L_{0}+1\right)\right)+q D_{0}\left(2 L_{0}+1\right) \\
\leq & D_{0}+q\left(1+2 D_{0}\left(2 L_{0}+1\right)\right) \leq\left(\delta^{-1} M+2\right)\left(1+2 D_{0}\left(2 L_{0}+1\right)\right)<M_{0} .
\end{aligned}
$$

Theorem 3.2 is proved. * 


\section{References}

[1] B. D. O. Anderson and J. B. Moore Linear optimal control Prentice-Hall, Englewood Cliffs, NJ 1971

[2] S. M. Aseev, K. O. Besov and A. V. Kryazhimskii Infinite-horizon optimal control problems in economics, Russ. Math. Surv. 67, (2012), 195-253

[3] S. Aubry and P. Y. Le Daeron The discrete Frenkel-Kontorova model and its extensions I Physica D 8 (1983), 381-422

[4] J. Baumeister, A. Leitao and G. N. Silva On the value function for nonautonomous optimal control problem with infinite horizon Systems Control Lett. 56, 188-196 (2007).

[5] J. Blot Infinite-horizon Pontryagin principles without invertibility J. Nonlinear Convex Anal. 10 (2009), 177-189.

[6] J. Blot and P. Cartigny Optimality in infinite-horizon variational problems under sign conditions J. Optim. Theory Appl. 106, 411-419 (2000).

[7] J. Blot and N. Hayek Sufficient conditions for infinite-horizon calculus of variations problems ESAIM Control Optim. Calc. Var. 5 (2000), 279-292.

[8] P. Cartigny and P. Michel On a sufficient transversality condition for infinite horizon optimal control problems Automatica J. IFAC 39 (2003), 1007-1010.

[9] D. Gale On optimal development in a multi-sector economy Review of Economic Studies 34, 1-18 (1967).

[10] N. Hayek Infinite horizon multiobjective optimal control problems in the discrete time case Optimization 60 (2011), 509-529.

[11] H. Jasso-Fuentes and O. Hernandez-Lerma Characterizations of overtaking optimality for controlled diffusion processes Appl. Math. Optim. 57 (2008), 349-369.

[12] A. Leizarowitz Infinite horizon autonomous systems with unbounded cost Appl. Math. and Opt. 13 (1985), 19-43.

[13] A. Leizarowitz and V. J. Mizel One dimensional infinite horizon variational problems arising in continuum mechanics Arch. Rational Mech. Anal. 106 (1989), 161-194.

[14] V. Lykina, S. Pickenhain and M. Wagner Different interpretations of the improper integral objective in an infinite horizon control problem J. Math. Anal. Appl. 340 (2008), 498-510.

[15] A. B. Malinowska, N. Martins and D. F. M. Torres Transversality conditions for infinite horizon variational problems on time scales Optim. Lett. 5 (2011), 41-53. 
[16] M. Marcus and A. J. Zaslavski The structure of extremals of a class of second order variational problems Ann. Inst. H. Poincare, Anal. non lineare 16, 593-629 (1999).

[17] L. W. McKenzie Turnpike theory Econometrica 44 (1976), 841-866.

[18] B. Mordukhovich Optimal control and feedback design of state-constrained parabolic systems in uncertainly conditions Appl. Analysis 90 (2011), 1075-1109

[19] B. Mordukhovich and I. Shvartsman Optimization and feedback control of constrained parabolic systems under uncertain perturbations Optimal Control, Stabilization and Nonsmooth Analysis, Lecture Notes Control Inform. Sci.(2004), Springer, 121-132 .

[20] E. Ocana and P. Cartigny Explicit solutions for singular infinite horizon calculus of variations SIAM J. Control Optim. 50(2012), 2573-2587.

[21] E. Ocana, P. Cartigny and P. Loisel Singular infinite horizon calculus of variations. Applications to fisheries management J. Nonlinear Convex Anal. 10 (2009), 157-176.

[22] S. Pickenhain, V. Lykina and M. Wagner On the lower semicontinuity of functionals involving Lebesgue or improper Riemann integrals in infinite horizon optimal control problems Control Cybernet. 37 (2008), 451-468.

[23] T. Prieto-Rumeau and O. Hernandez-Lerma Bias and overtaking equilibria for zero-sum continuous-time Markov games Math. Methods Oper. Res. 61 (2005), 437-454.

[24] A.M. Rubinov Economic dynamics J. Soviet Math. 26 (1984), 1975-2012.

[25] P. A. Samuelson A catenary turnpike theorem involving consumption and the golden rule American Economic Review 55 (1965), 486-496.

[26] A. J. Zaslavski Ground states in Frenkel-Kontorova model Math. USSR Izvestiya 29 (1987), 323-354.

[27] A. J. Zaslavski Turnpike properties in the calculus of variations and optimal control Springer, New York, 2006.

[28] A. J. Zaslavski Turnpike results for a discrete-time optimal control system arising in economic dynamics Nonlinear Analysis 67, (2007) 2024-2049.

[29] A. J. Zaslavski Two turnpike results for a discrete-time optimal control system Nonlinear Analysis 71, (2009) 902-909 .

[30] A. J. Zaslavski Structure of solutions of variational problems. SpringerBriefs in Optimization, Springer, New York 2013. 
[31] A. J. Zaslavski Necessary and sufficient conditions for turnpike properties of solutions of optimal control systems arising in economic dynamics Dynamics of Continuous, Discrete and Impulsive Systems, Ser. B Appl. Algorithms 20 (2013), 391-420.

[32] A. J. Zaslavski and A. Leizarowitz Optimal solutions of linear control systems with nonperiodic integrands Mathematics of Operations Research 22 (1997), 726746.

DOI: $10.7862 /$ rf.2015.12

Alexander J. Zaslavski

email: ajzasl@tx.technion.ac.il

Department of Mathematics,

Technion-Israel Institute of Technology,

32000, Haifa, Israel

Received 03.06.2014 